\title{
When food can make the difference: The case of elvitegravir-based co- formulation
}

\author{
Dario Cattaneo, Sara Baldelli, Davide Minisci, Paola meraviglia, Emilio Clementi, Massimo Galli, \\ Cristina Gervasoni
}

ABSTRACT

Stribild should be administered under fed conditions to optimize drugs exposure. Here we assessed to what extent this advice is applied in the real life scenario by therapeutic drug monitoring in 75 HIV- infected patients given Stribild-based antiretroviral therapy. Fifty-three percent of our patients took Stribild at lunch/supper time, $23 \%$ in the morning with breakfast, and $24 \%$ middle in the morning or late in the evening. Twelve out of the 75 patients had unquantifiable elvitegravir concentrations, whereas in the remaining the levels were largely distributed. Wide inter-individual variability in the tenofovir, cobicistat and darunavir trough concentrations was also observed.

In real life settings a significant proportion of patients took Stribild without food, namely in the mid- morning or late in the evening. This resulted in a wide inter-individual variability of antiretroviral drug trough concentrations. To avoid the risk for patients to experience suboptimal drug exposure, it is important that health professionals more convincingly advise their patients to take Stribild in fed conditions. On the other hand, the role of patient education and patient responsibility to correctly take the therapy should not be underestimated.

TEXT

In the product monograph of the fixed-dose combination containing elvitegravir, cobicistat, tenofovir and emtricitabine (Stribild) it is recommended that the formulation should be administered under fed conditions to optimize drugs exposure ( Stribild monograph, 2016; Ramanathan et al., 2011). Here we assessed to what extent this advice is applied in the real life scenario by measuring elvitegravir, tenofovir and cobicistat plasma trough concentrations in HIV-infected patients given Stribild alone or as part of antiretroviral therapy as per their daily routine practice. Emtricitabine concentrations were not measured because therapeutic monitoring of this drug is not considered a useful tool to optimize drug dosing of HIV-infected patients in the clinical practice. In this retrospective analysis we included all consecutive HIV- infected patients from our database fulfilling the following conditions: a) treated with Stribild for at least one month, b) with at least one request for therapeutic drug monitoring (TDM) of elvitegravir and tenofovir plasma trough concentrations, c) no clinical evidence of gastrointestinal impairment and 4) not given drugs known to affect elvitegravir or tenofovir pharmacokinetics. Collected blood samples had to be taken $24 \mathrm{~h}$ after the last drug intake (a time window of `20min was considered acceptable), immediately before drug administration, to ensure that these samples can be considered as trough concentrations.

The main demographic, biochemical and clinical data were recorded for each patient, together with detailed information on the time of blood drawing and the time of last drug intake (no information on the type of food eaten by the patients were available). Elvitegravir, tenofovir, cobicistat and darunavir trough concentrations were assessed by liquid chromatography tandem mass spectrometry methods developed and validated in our lab (lower limit of quantification, LOQ: 25, 10, 5 and $100 \mathrm{ng} / \mathrm{mL}$ for elvitegravir, tenofovir, cobicistat and darunavir, respectively). Data were expressed as median [interquartile range]. Written informed consent for medical procedures/interventions performed for routine treatment purposes was collected for each patient. Given the retrospective, observational nature of this research, no formal approval from the local ethics committee was required according to national legislation.

Seventy-five HIV-infected adult patients were included in this study. These patients had median age of 45 [38-50] years, were mainly Caucasian (75\%), male (80\%) and were at a median of 173 [56-356] days of therapy with Stribild. Thirty-seven out of the 75 patients were also given a protease inhibitor, namely darunavir $(n=35)$ or atazanavir $(n=2)$. All patients had good immunological status (CD4 cell count: 487 [354-697] cells/mL); fourteen out of the 75 patients had detectable viral load ( $>37$ copies $/ \mathrm{mL}$ ) at the time of TDM: of these, 6 patients were at more than 6 months of therapy with Stribild. None of the patients had clinical signs of liver (serum alanine aminotrasferase: 31 [21-41] IU/mL) or kidney (serum creatinine: 0.9 [0.8-1.1] mg/dL) dysfunction. No obese patients were included in the study (body weight: 70 [65-77] kg; body mass index: 23.6 [21.6-25.2] kg/ Thirty-six percent of our patients took Stribild in the evening with food, and the remaining were distributed as follows: $23 \%$ in the morning with breakfast, $9 \%$ middle in the morning, $17 \%$ at lunchtime and $15 \%$ late in the evening. Twelve out of the 75 patients (16\%) had elvitegravir concentrations below the LOQ of the method, whereas in the remaining the levels were largely distributed, ranging from 50 to $2311 \mathrm{ng} / \mathrm{mL}$ (Fig. 1, left panel). All patients with unquantifiable elvitegravir levels took Stribild under fasting conditions. One of these patients had detectable viral load ( 975 copies $/ \mathrm{mL}$ ). Wide inter-individual variability in the tenofovir levels was also observed, ranging from 21 to $950 \mathrm{ng} / \mathrm{mL}$ (Fig. 1, middle panel). Tenofovir levels were significantly lower in patients with elvitegravir concentrations < LOQ as compared with patients with quantifiable elvitegravir concentrations (tenofovir trough: 52 [27-81] vs. 137 [105-211] ng/mL; p < 0.01). Same findings were observed also for cobicistat (Fig. 1, right panel), with 18 out of the 75 patients having drug concentrations $<$ LOQ. Of these, patients given Stribild with darunavir had drug concentration significantly lower compared with values mea- sured in patients with quantifiable cobicistat levels (darunavir trough: 225 [150-266] vs. 2292 [1435-3908] ng/mL; p < 0.01). Positive and significant correlations were found between elvitegravir and cobicistat concentrations $(\mathrm{r}=0.68, \mathrm{p}<0.01)$, as well as between darunavir and cobicistat concentrations $(\mathrm{r}=$ $0.56, \mathrm{p}<0.01$ ). No associations between drugs plasma trough concen- trations and clinical covariates (namely serum transaminases, serum creatinine levels, body weight or body mass index) were observed. 
Six out of the 12 patients with elvitegravir concentrations < LOQ had more than 1 TDM assessment. In these patients the concentrations of elvitegravir, tenofovir and cobicistat measured in the second TDM resulted greatly higher compared with the first TDM assessment (Fig. 2). At the second TDM assessment the six patients were taking Stribild with food.

Here we have documented that in a real life context a significant proportion of patients took Stribild in fasting conditions, namely in the mid-morning or late in the evening. This resulted in a wide inter-individual variability of elvitegravir and tenofovir plasma trough concentrations. Worthy of mention, in some patients such concentrations resulted below the protein- adjusted set for both drugs at around 50ng/mL (Ramanathan et al., 2016; Kearney et al., 2004; Gervasoni et al., 2013; Flynn et al., 2011). Such pharmacokinetic variability was not related to clinical characteristics of the enrolled patients, such as renal or liver function, body weight. Rather, it is likely that the type of food consumed varied between patients, and this may have lead to differences in the plasma drug trough concen- trations. Indeed, the highest concentrations are expected in patients given lipophilic drugs with a high-fat meal due to increased drug solubilization in fatty food milieu. This may be the case for elvitegravir and cobicistat (partition coefficient: 4.5 and 4.3, respectively) and less likely for tenofovir (partition coeffi- cient: 1.25) (Stribild monograph, 2016). Conversely, the lowest drug concentrations were measured in patients given Stribild in fasting conditions. In view of that, it can be hypothesized that the intake of Stribild without food may a) reduce drugs solubility, b) decrease the resident time of the drugs in the stomach, and/or c) limit the disintegration of the pharmaceutical formulation, all conditions ultimately resulting in reduced systemic drug bio- availability. This hypothesis is indirectly supported also by the fact that the concentrations of tenofovir measured in patients given Stribild in fed conditions were comparable to those measured in patients given tenofovir not co-formulated with elvitegravir (Adams et al., 2012). Furthermore, this evidence suggests that the role of food is not significant only for elvitegravir pharmacokinetics, as recently documented (Shiomi et al., 2014), but it can potentially affect also tenofovir and cobicistat exposure. The latter condition may become particularly relevant when darunavir is co-administered in order to reinforce the antiretro- viral therapy, because the cobicistat-related boosting effect is lost, eventually resulting in subtherapeutic darunavir concentrations. Indeed, the reported darunavir protein-adjusted mutant viruses, respectively (Prezista monograph, 2016; Delaugerre et al., 2007).

The present study was not designed to assess whether the observed variability of elvitegravir and tenofovir pharmacokinetics related to food could eventually impact on patient outcome. For most patients we had only one TDM assessment, therefore we could only speculate that some of them may have transitorily exposed to subtherapeutic drugs concentrations.

The study, given its retrospective design, has some limitations. Firstly, we have no data on emtricitabine concentrations. Moreover, we were not able to assess the contribution of the type of food eaten by the patients to the observed inter-individual variability in the drugs trough concentrations. Finally, the possibility that our results could have been affected at least in part by non-adherence and inaccurate patients self-reporting on the prior dose time cannot be fully ruled out.

In conclusion, the role of food in improving Stribild bioavailability is underestimated in real life settings. Since this condition may increase the risk for patients to experience suboptimal drug exposure, it is important that all health professionals (physicians, pharmacists, nurses) more convincing- ly advise their patients to take Stribild in fed conditions. On the other hand, the key role of patient education and patient responsibility to be fully adherent with the instructions provided by healthcare professionals on how to correctly take the therapy should not be underestimated.

Conflict of interest

All the authors declare non conflict of interest.

Authors contribution

All the authors have significantly contributed to the conception and design of the study, interpretation of the data, drafting the manuscript, approved the final version of the paper.

Funding

This is an independent, retrospective study carried out as part of the routine management of patients. This research received no specific grant from any funding agency in the public, commercial, or not-for-profit sectors.

\section{References}

Adams, J.L., Greener, B.N., Kashuba, A.D., 2012. Pharmacology of HIV integrase inhibitors. Curr. Opin. HIV AIDS 7, 390-400.

Delaugerre, C., Mathez, D., Peytavin, G., Berthé, H., Long, K., Galperine, T., de Truchis, P., 2007. Key amprenavir resistance mutations counteract dramatic efficacy of darunavir in highly experienced patients. AIDS 2, 1210-1213.

Flynn, P.M., Mirochnick, M., Shapiro, D.E., Bardeguez, A., Rodman, J., Robbins, B., Huang, S., Fiscus, S.A., Van Rompay, K.K., Rooney, J.F., Kearney, B., Mofenson, L. M., Watts, D.H., Jean-Philippe, P., Heckman, B., Thorpe Jr, E., Cotter, A., Purswani, M., PACTG 394 Study Team, 2011. Pharmacokinetics and safety of single-dose tenofovir disoproxil fumarate and emtricitabine in HIV-1-infected pregnant women and their infants. Antimicrob. Agents Chemother. 55, 5914-5922. 
Kearney, B.P., Flaherty, J.F., Shah, J., 2004. Tenofovir disoproxil fumarate: clinical pharmacology and pharmacokinetics. Clin. Pharmacokinet. 43, 595-612.

PREZISTA Monograph: Available at https://www.janssen.com/canada/sites/ www_janssen_com_canada/files/product/pdf/prez09182014cpm2_nc.pdf. (accessed 26. 06. 16.).

Ramanathan, S., Mathias, A.A., German, P., Kearney, B.P., 2011. Clinical pharmacokinetic and pharmacodynamic profile of the HIV integrase inhibitor elvitegravir. Clin. Pharmacokinet. 50, 229-244. Ramanathan, S., Custodio, J.M., Wei, X., Wang, H., Fordyce, M., Dave, A., Ling, K.H., Szwarcberg, J., Kearney, B.P., 2016. Pharmacokinetics of co-formulated elvitegravir/cobicistat/emtricitabine/tenofovir disoproxil fumarate following switch from efavirenz/emtricitabine/tenofovir disoproxil fumarate in healthy subjects. J. Acquir. Immune Defic. Syndr. Epub ahead of print.

Shiomi, M., Matsuki, S., Ikeda, A., Ishikawa, T., Nishino, N., Kimura, M., Irie, S., 2014. Effects of a protein-rich drink or a standard meal on the pharmacokinetics of

elvitegravir, cobicistat, emtricitabine and tenofovir in healthy Japanese male subjects: a randomized, three-way crossover study. J. Clin. Pharmacol. 54, 640-648.

STRIBILD Monograph. Available at http://www.gilead.ca/pdf/ca/stribild_pm_english.pdf. accessed (26.06.16.). 